\title{
Influence of shape of quantum dots on their far-infrared absorption
}

\author{
Ingibjörg Magnúsdóttir and Vidar Gudmundsson \\ Science Institute, University of Iceland, Dunhaga 3, IS-107 Reykjavik, Iceland
}

(June 22, 1999)

\begin{abstract}
We investigate the effects of the shape of quantum dots on their far-infrared absorption in an external magnetic field by a model calculation. We focus our attention on dots with a parabolic confinement potential deviating from the common circular symmetry, and dots having circular doughnut shape. For a confinement where the generalized Kohn theorem does not hold we are able to interprete the results in terms of a mixture of a center-of-mass mode and collective modes reflecting an excitation of relative motion of the electrons. The calculations are performed within the time-dependent Hartree approximation and the results are compared to available experimental results.

73.20.Dx, 71.70.Di, 73.20.Mf
\end{abstract}

Typeset using REVTEX 


\section{INTRODUCTION}

Ever since the discovery that far-infrared radiation (FIR) can only be used to excite center-of-mass modes of electrons parabolically confined in circular quantum dots, the generalized Kohn theorem, 3 ingenious ways have been thought of to modify the confinement in order to excite internal modes. The modes caused by relative motion of the electrons would allow for exploration of interaction and general many-body effects. It was demonstrated that in circular dots with a slight radial deviation from the parabolic confinement states with few electrons have a unique far-infrared spectrum. Dots with a higher number of electrons $(N>10)$ commonly only show a simpler structure of Bernstein modes $\omega$ and an energy shift 6 . Early on, it came clear that a certain anticrossing behavior discovered in the higher one of the two Kohn's modes measured 8 for quantum dots assumed to be circular is indeed a signature of a slight square deformation of the electron system.9.6 The far-infrared absorption of strictly square-shaped quantum dots with hard walls has been modelled by exact numerical diagonalization for two electrons, 10, 11 and by a real-time simulation of the density oscillations described by a local spin-density approximation (LSDA) for many electrons. 12 The generalized Kohn theorem has been further extended to describe elliptid 13 quantum dots and related three-dimensional structures, ellipsoids.4

The production of arrays of isolated angularly deformed quantum dots has been hampered by technical difficulties. The little there is of experimental results from FIR absorption measurements on quantum dots with angular deviation it has not found its way into the general physics literature but is to be found as thesis work $15-17$

The FIR absorption of single quantum rings has been studied in relatively large systems with many electrons, 18,19 but recently small quantum dots with few electrons and a hole through their center have been produced and measured.20

In this publication we model the FIR absorption of single quantum dots with a slight elliptic or square deviation assuming the radial confinement to be parabolic. The model is general enough to be applicable for most angular deviations of the confinement. In a circular quantum dot the electron-electron interaction does not break the angular symmetry, but in our model it can strongly modify the shape of the dot as soon as the circular symmetry is broken by the initial confinement. We apply our model to the FIR absorption of an elliptic dot in order to verify that it produces the results expected by Kohn's theorem.13 The calculated absorption spectra for a square deviated quantum dot show both familiar results for a weak deviation, 99 and effects reflecting internal electron motion that have not yet been observed in experiments.

In addition, we calculate the FIR absorption for a small dot with few electrons with its center removed to compare with the results of A. Lorke.20

\section{MODEL}

As we allow for a very general angular shape of the quantum dot neither the total angular momentum nor the angular momentum of the effective single particle states in a mean field approach is conserved. The Coulomb interaction thus 'mixes' up all elements of the functional basis used and we limit ourselves to the Hartree approximation (HA) in order 
to be able to calculate the absorption. The quantum dot is modelled with the confinement potential

$$
V_{\text {conf }}(\mathbf{r})=\frac{1}{2} m^{*} \omega_{0}^{2} r^{2}\left[1+\sum_{p=1}^{p_{\max }} \alpha_{p} \cos (2 p \phi)\right],
$$

representing an elliptic confinement when $\alpha_{1} \neq 0$ and $\alpha_{p}=0$ for $p \neq 1$, and a square symmetric confinement when $\alpha_{2} \neq 0$ and $\alpha_{p}=0$ for $p \neq 2$. We use the Darwin-Fock basis; the eigenfunctions of the circular parabolic confinement potential in which the natural length scale, $a$, is given by

$$
a^{2}=\frac{\ell^{2}}{\sqrt{1+4\left(\frac{\omega_{0}}{\omega_{c}}\right)^{2}}}, \quad \ell^{2}=\frac{\hbar c}{e B},
$$

where $\omega_{c}=e B / m^{*} c$ is the cyclotron frequency of an electron with effective mass $m^{*}$ in a perpendicular homogeneous magnetic field $B$. The states are labelled by the radial quantum number $n_{r}$ and the angular quantum number $M .6$ The single electron spectrum of these states is shown in Fig. 11.

To evaluate the FIR response of the quantum dot, the external potential is assumed to have the form

$$
\phi^{e x t}(\mathbf{r}, t)=\phi_{0} r e^{i\left(N_{p} \phi-(\omega+i \eta) t\right)},
$$

where $N_{p}= \pm 1$ and $\eta \rightarrow 0^{+}$, representing a spatially constant external electric field. The FIR response is found by a self-consistent method in the linear response regime; the timedependent Hartree approximation. The power absorption is then given by

$$
\mathcal{P}(\omega) \propto \omega \Im\left\{\sum_{\alpha, \beta} f^{\beta \alpha}(\omega)\left|\left\langle\beta\left|\phi^{s c}\right| \alpha\right\rangle\right|^{2}\right\},
$$

where

$$
f^{\beta \alpha}(\omega)=\frac{1}{\hbar}\left\{\frac{f_{\alpha}^{0}-f_{\beta}^{0}}{\omega+\left(\omega_{\alpha}-\omega_{\beta}\right)+i \eta}\right\}
$$

and $f^{0}$ is the equilibrium Fermi distribution. Self-consistency is obtained by calculating linear response not to the external perturbation, $\phi^{e x t}$, but rather to the total (self-consistent) potential $\phi^{s c}=\phi^{e x t}+\phi_{H}^{i n d}$, where $\phi_{H}^{i n d}$ is the induced Hartree potential. For a circular quantum dot, the parabolic potential has proven to be a realistic approximation in many cases. For such a circular harmonic dot, the dipole selection rule for the center-of-mass angular momentum is $\Delta M=N_{p}$ and the resonance frequencies are given by

$$
\omega_{ \pm}=\frac{1}{2}\left(\Omega+N_{p} \omega_{c}\right), \quad \Omega=\left(\omega_{c}^{2}+4 \omega_{0}^{2}\right)^{1 / 2} .
$$

For an anisotropic harmonic confinement, the selection rule is still $\Delta M= \pm 1$, but there is absorption into both $\omega_{+}$and $\omega_{-}$for each polarization. The resonance frequencies are then given by

$$
\omega_{ \pm}^{2}=\frac{\omega_{x}^{2}+\omega_{y}^{2}+\omega_{c}^{2} \pm\left[\omega_{c}^{4}+2 \omega_{c}^{2}\left(\omega_{x}^{2}+\omega_{y}^{2}\right)+\left(\omega_{x}^{2}-\omega_{y}^{2}\right)^{2}\right]^{1 / 2}}{2} .
$$

where $\omega_{x}$ and $\omega_{y}$ are the resonances at $B=0$ T, 1, 13 fulfilling $\omega_{x}=\left(1+\alpha_{1}\right)^{1 / 2} \omega_{0}$ and $\omega_{y}=\left(1-\alpha_{1}\right)^{1 / 2} \omega_{0}$ in our model. 


\section{RESULTS}

In the calculations reported here we use GaAs parameters, the effective mass $m^{*}=$ $0.067 m_{0}$ and the dielectric constant $\kappa=12.4$. The FIR absorption of a quantum dot with an elliptic deviation is seen in Fig. 2. The induced density for the oscillation modes was used to confirm that only center-of-mass modes are excited and the dispersion with respect to $B$ can be reproduced as a simple difference of the dipole active transfers in the DarwinFock energy spectrum for one electron in a parabolic elliptic confinement. The oscillation strengths do comply with known analytic expressions.13 Interestingly, the external circular polarized electric field leads to linear oscillations of the electron system parallel to the minor and major axis of the contours of the elliptic confinement potential.

Figure 3 shows the Darwin-Fock energy for a single electron in a confining potential with square deviation. Clearly visible is the anticrossing of the $\left(M, n_{r}\right)=(+1,0)$ and the state $(-3,0)$ that was found to be visible in the FIR absorption of quantum dots which were clearly of square shape in an electron micrograph.8.6 This anticrossing is present in the calculated absorption shown in Fig. 4 and 5 for two electrons in a dot. For this relative large deviation $\left(\alpha_{2}=0.2\right.$ or 0.4$)$ from circular symmetry the absorption at $B=0$ for both circular polarizations $\left(N_{p}= \pm 1\right)$ is split in two or more peaks. This is in contrast to the Darwin-Fock energy diagram in Fig. 3 from which we have to conclude that the lowest energy center-of-mass mode should be unsplit at $B=0$. Certainly it has to be kept in mind that the states in Fig. 3 can not be assigned any unique quantum numbers, $n_{r}$ and $M$. The evolution from one absorption peak to many at higher deviation is seen in Fig. 6. Observation of the induced density shows that all the modes are a mixture of center-of-mass and relative modes to different extent here. Some peaks can be identified with depolarization shifted transitions in the interacting Hartree energy spectrum while others are very close to transitions in the noninteracting Darwin-Fock diagram reflecting center-of-mass modes.

In Fig. 7 the FIR absorption is shown for three electrons, $(N=3)$. A splitting or anticrossing occurs in the absorption for $N=3$ and $N_{p}=-1$ whereas it does not for $N=2$. This striking behavior can be understood from the Darwin-Fock energy diagram for three electrons (Fig. 8). The splitting can be identified as a transition from the third energy level to the fourth and fifth with a depolarization shift, showing its many-body character. This behavior can not be seen for $N=2$, since the third level is then not occupied.

Generally the lower energy branch (seen in $N_{p}=-1$ polarization in circular dots) is very stable against splitting, especially for dots with many electrons. One exception is a dot with some kind of a hole through its center. Such dots have been grown as self-organized InAs rings embedded into a GaAs/AlGaAs heterostructure. Here we try to model simply the qualitative behavior of such dots by using GaAs parameters and by replacing the hole with a repulsive Coulomb center in the middle of the dot. The confinement of the electrons is thus not of the same type as was used to study the far-infrared absorption of a finite 2DEG with an embedded impurity.21 The calculated absorption is seen in Fig. 9 for two and twelve electrons in the quantum ring. To understand the absorption one has to consider the Darwin-Fock energy spectrum of the Hartree interacting electrons in the ring shown in Fig. 10. In the case of two electrons in the ring the emptying of states with low angular quantum number $M$ close to the center with increasing magnetic field $B$ results in low energy 'side branches' with polarization $N_{p}=+1$ to the $N_{p}=-1$ branch. The magnetic 
length, $a$, decreases with increasing $B$, causing the electrons to 'see' in a sence more of the repulsice Coulomb center (and each other), resulting in the emptying of states of low $M$. It is our suggestion that A. Lorke is observing this same effect in his experiment.20 It should be mentioned here that the electron density in our calculation at the center of the ring is low for $B=0$ and vanishes as $B$ increases. In the case of twelve electrons screening effects and oscillating structure reminiscent of emptying of Landau bands is quite clear in the Darwin-Fock energy diagram, but again emptying of the $M=0$ state at $B=4 \mathrm{~T}$ is visible in the absorption together with restructuring of the states with high $M$ at the outer edge.

\section{SUMMARY}

We have used a general two-dimensional multipolar expansion of the confinement potential of a single quantum dot to study the effects of shape on its properties. We have shown that the far-infrared absorption spectrum of quantum dots with few electrons strongly reflects their shape and exact number of electrons if the confinement potential does not allow the application of Kohn's theorem. Elliptic dots have two low energy peaks of equal strength at $B=0$, whereas, dots with slight square symmetry reveal only one peak. This behavior is connected to the different effects of shape on the $\left(M, n_{r}\right)=( \pm 1,1)$ degeneracy of circular dots at $B=0$. We find that this degeneracy can be lifted by the activation of relative modes in the far-infrared absorption spectrum of square shaped dots with increased deviation from the circular form.

In quantum rings we observe a shift of occupation of the states labelled by the angular momentum quantum number $M$ to higher values with increasing magnetic field. This is essentially caused by the changing ratio of the magnetic length, $a$, and the dots radii. We identify structures seen in experiments with this effect.20

The Hartree approximation has been used here rather than the more desirable HartreeFock approximation in some unrestricted version, in order to manage the size of the absorption part of the calculation measured in GBytes and CPU-time. On the other hand, the HA can be expected to give a valid description of a system whose modes of excitation are mainly of the center-of-mass type.

\section{ACKNOWLEDGMENTS}

This research was supported by the Icelandic Natural Science Foundation and the University of Iceland Research Fund. 


\section{REFERENCES}

${ }^{1}$ P. A. Maksym and T. Chakraborty, Phys. Rev. Lett 65, 108 (1990).

${ }^{2}$ L. Brey, N. Johnson, and B. Halperin, Phys. Rev. B 40, 10647 (1989).

${ }^{3}$ P. Bakshi, D. Broido, and K. Kempa, Phys. Rev. B 42, 7416 (1990).

${ }^{4}$ D. Pfannkuche, V. Gudmundsson, P. Hawrylak, and R. Gerhardts, Solid-State Electronics 37, 1221 (1994).

${ }^{5}$ V. Gudmundsson et al., Phys. Rev. B 51, 17744 (1995).

${ }^{6}$ V. Gudmundsson and R. Gerhardts, Phys. Rev. B 43, 12098 (1991).

${ }^{7}$ Z. L. Ye and E. Zaremba, Phys. Rev. B 50, 17217 (1994).

${ }^{8}$ T. Demel, D. Heitmann, P. Grambow, and K. Ploog, Phys. Rev. Lett. 64, 788 (1990).

${ }^{9}$ D. Pfannkuche and R. Gerhardts, Phys. Rev. B 44, 13132 (1991).

${ }^{10}$ R. Ugajin, Phys. Rev. B 51, 10714 (1995).

${ }^{11}$ R. Ugajin, Phys. Rev. B 53, 6963 (1996).

${ }^{12}$ A. Puente and L. Serra, Oscillation modes of two-dimensional nanostructures within the time-dependent local-spin-density approximation, cond-mat/9905288, 1999.

${ }^{13}$ S. K. Yip, Phys. Rev. B 43, 1707 (1991).

${ }^{14}$ Q. P. Li et al., Phys. Rev. B 43, 5151 (1991).

${ }^{15}$ M. Hochgräfe, Master's thesis, University of Hamburg, Germany, 1998.

${ }^{16}$ E. Vasiliadou, Ph.D. thesis, University of Hamburg, Germany, 1995.

${ }^{17}$ C. Dahl, Ph.D. thesis, Ludwig-Maximilians-Universität, München, Germany, 1992.

${ }^{18}$ E. Zaremba, Phys. Rev. B 53, R10512 (1996).

${ }^{19}$ A. Emperador et al., Phys. Rev. B 59, in press (1999).

${ }^{20}$ A. Lorke and R. J. Luyken, Physica B 256-258, 424 (1998).

${ }^{21}$ V. Gudmundsson and Á. S. Loftsdóttir, Phys. Rev. B 50, 17433 (1994). 


\section{FIGURES}

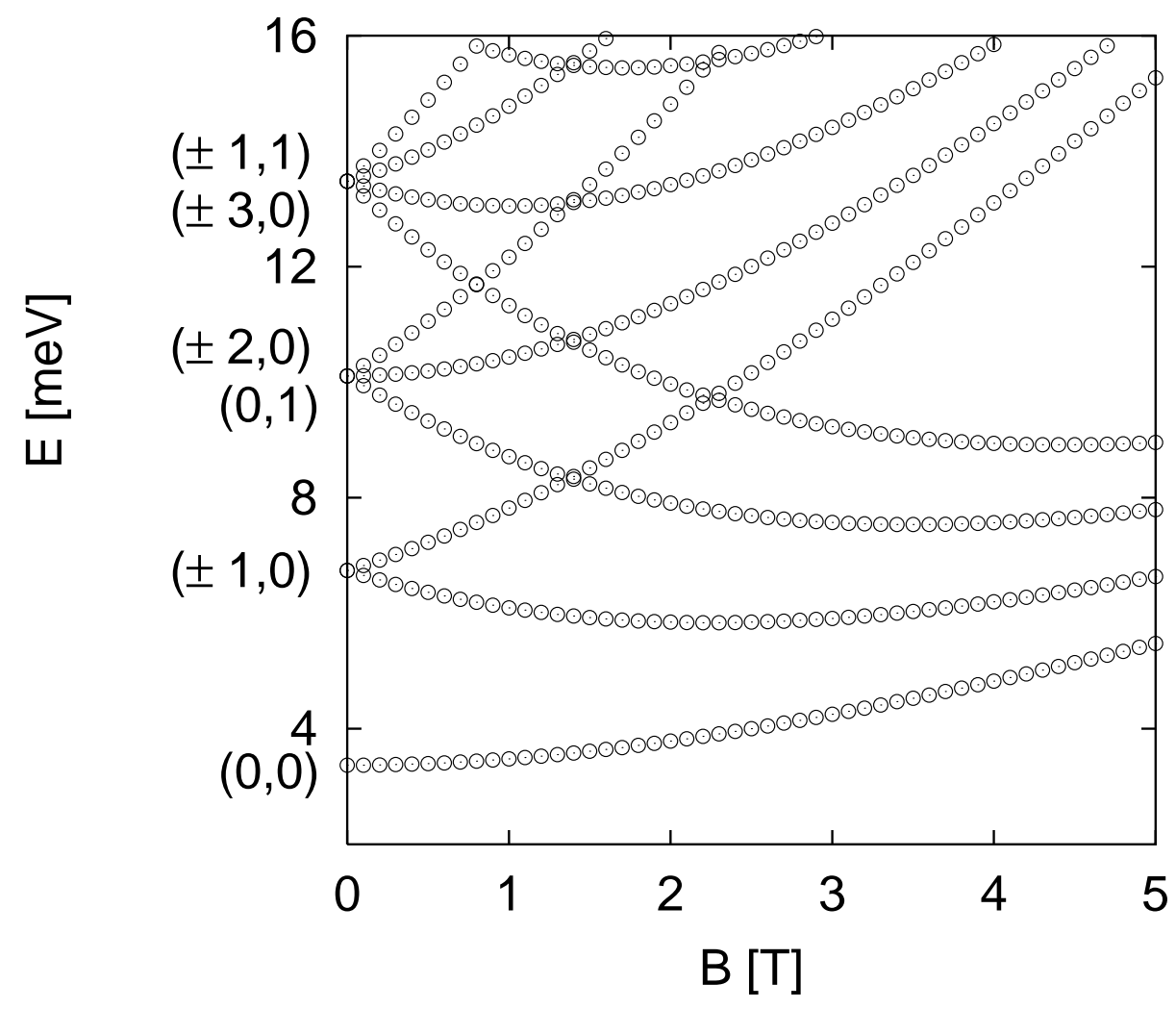

FIG. 1. The Darwin-Fock energy diagram of a single electron in a circular parabolic quantum dot. At $B=0$, we have the familiar two-dimensional harmonic oscillator. The states at $B=0$ are labelled with $\left(M, n_{r}\right)$. 

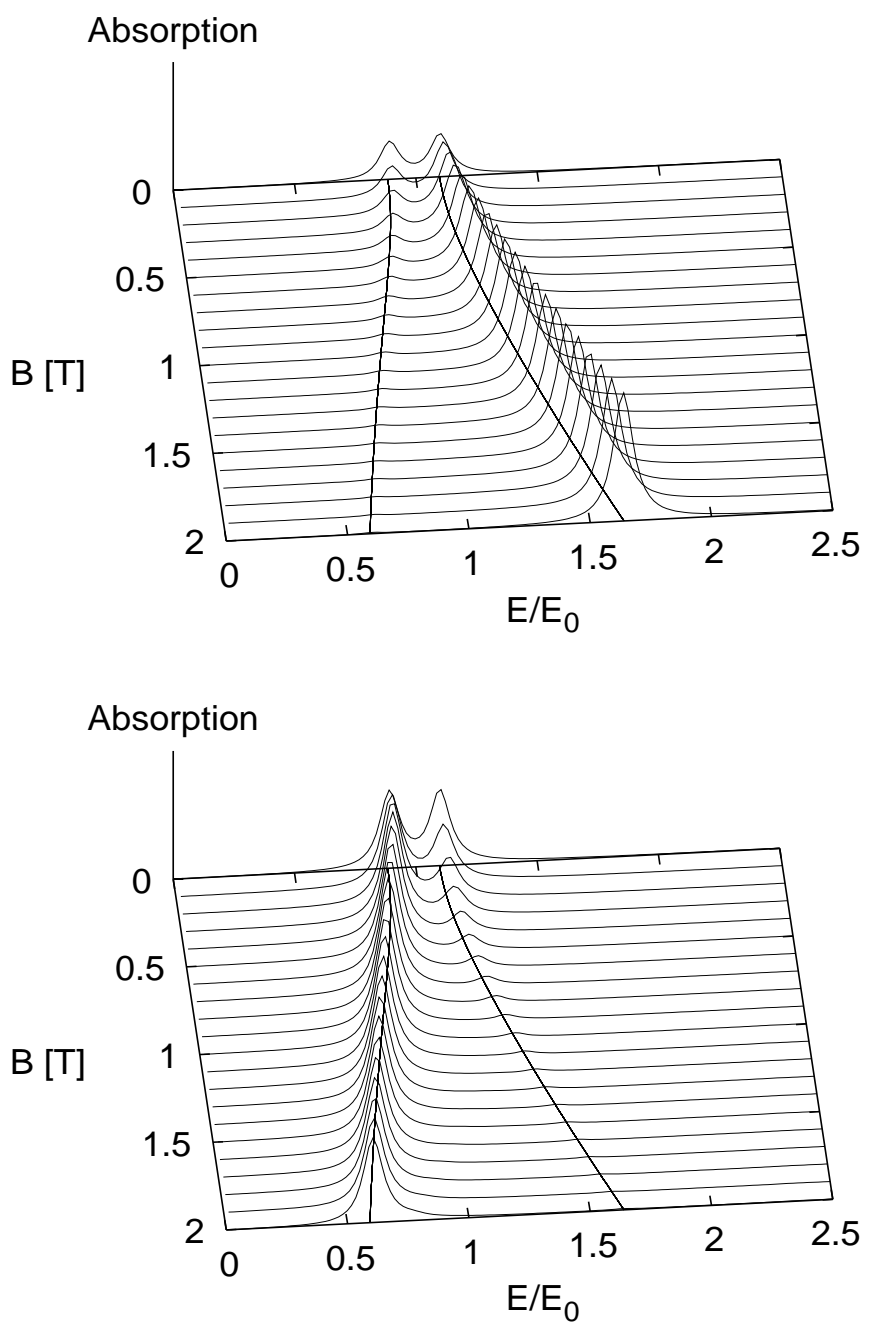

FIG. 2. The far-infrared absorption for $N_{p}=+1$ (above) and $N_{p}=-1$ (below) in the case of a two electron elliptic quantum dot $\left(\alpha_{1}=0.20\right)$. The solid lines indicate the dispersion relation for the absorption, with $\omega_{x} / \omega_{0}=1.10$ and $\omega_{y} / \omega_{0}=0.89$. 


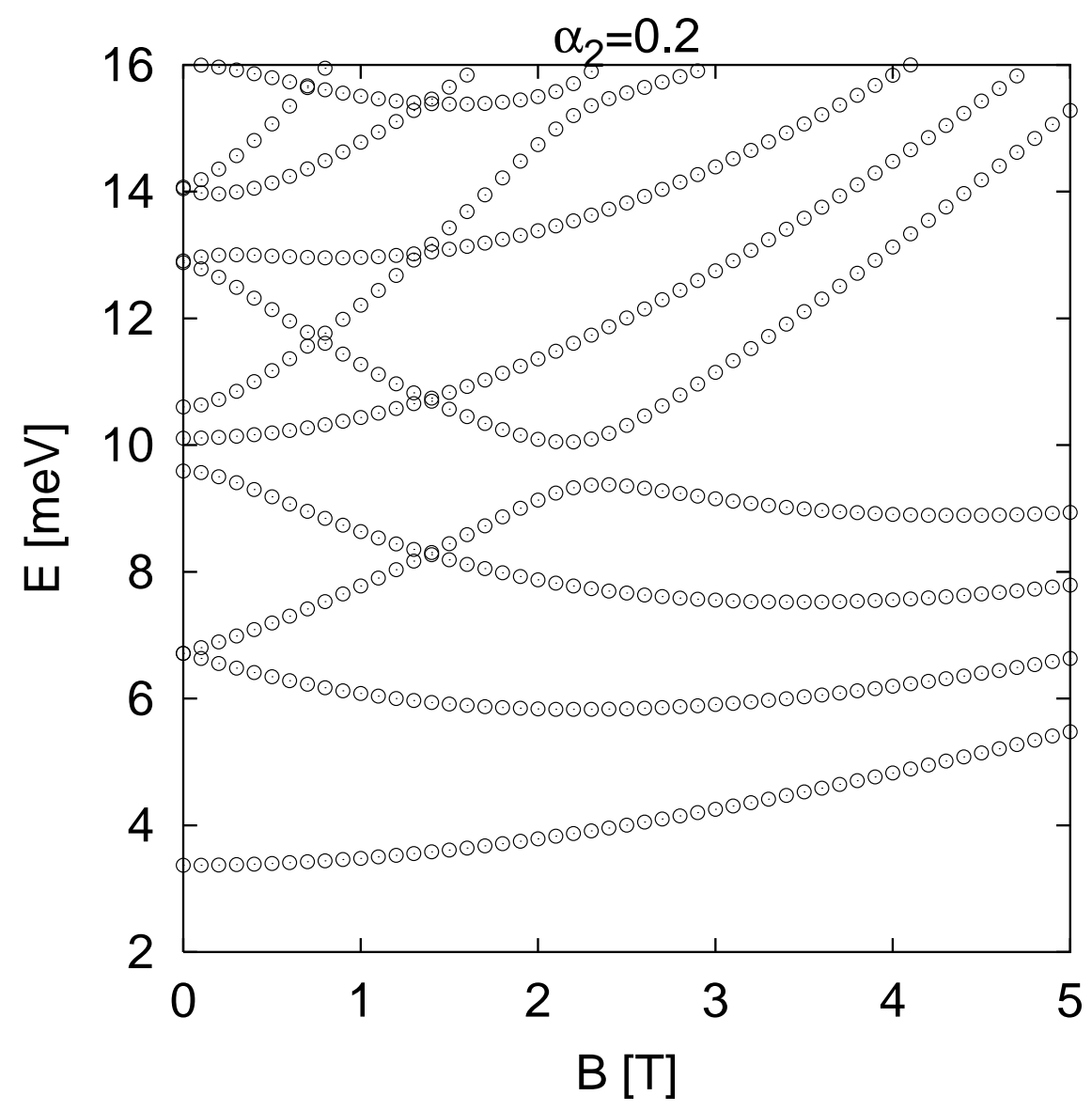

FIG. 3. The Darwin-Fock energy diagram of a single electron in a square symmetric quantum dot with $\alpha_{2}=0.20$. The angular part of $V_{\phi}, \cos (4 \phi)$, couples states in the circular parabolic potential for which $\Delta M= \pm 4$. Degeneracy of states at $B=0$ fulfilling $\Delta M= \pm 4$ is lifted. An anticrossing occurs at $B \approx 2.3 T$ due to coupling of the accidentally degenerate states $(+1,0)$ and $(-3,0)$ at this magnetic field. 

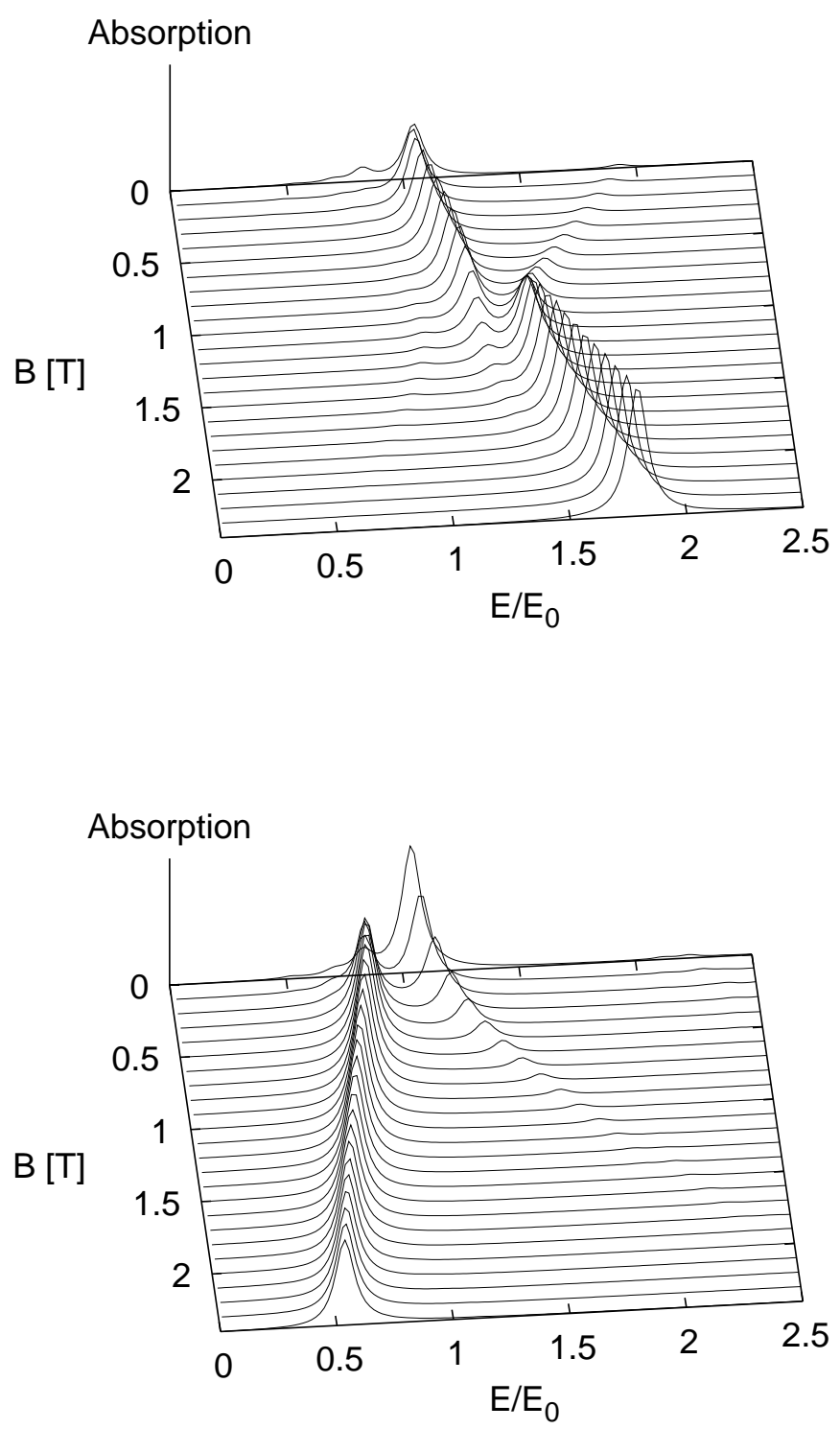

FIG. 4. The far-infrared absorption of a two electron square symmetric quantum dot $\left(\alpha_{2}=0.20\right)$ for $N_{p}=+1$ (above) and $N_{p}=-1$ (below). $E_{0}=3.37 \mathrm{meV}, T=1.0 \mathrm{~K}$. 

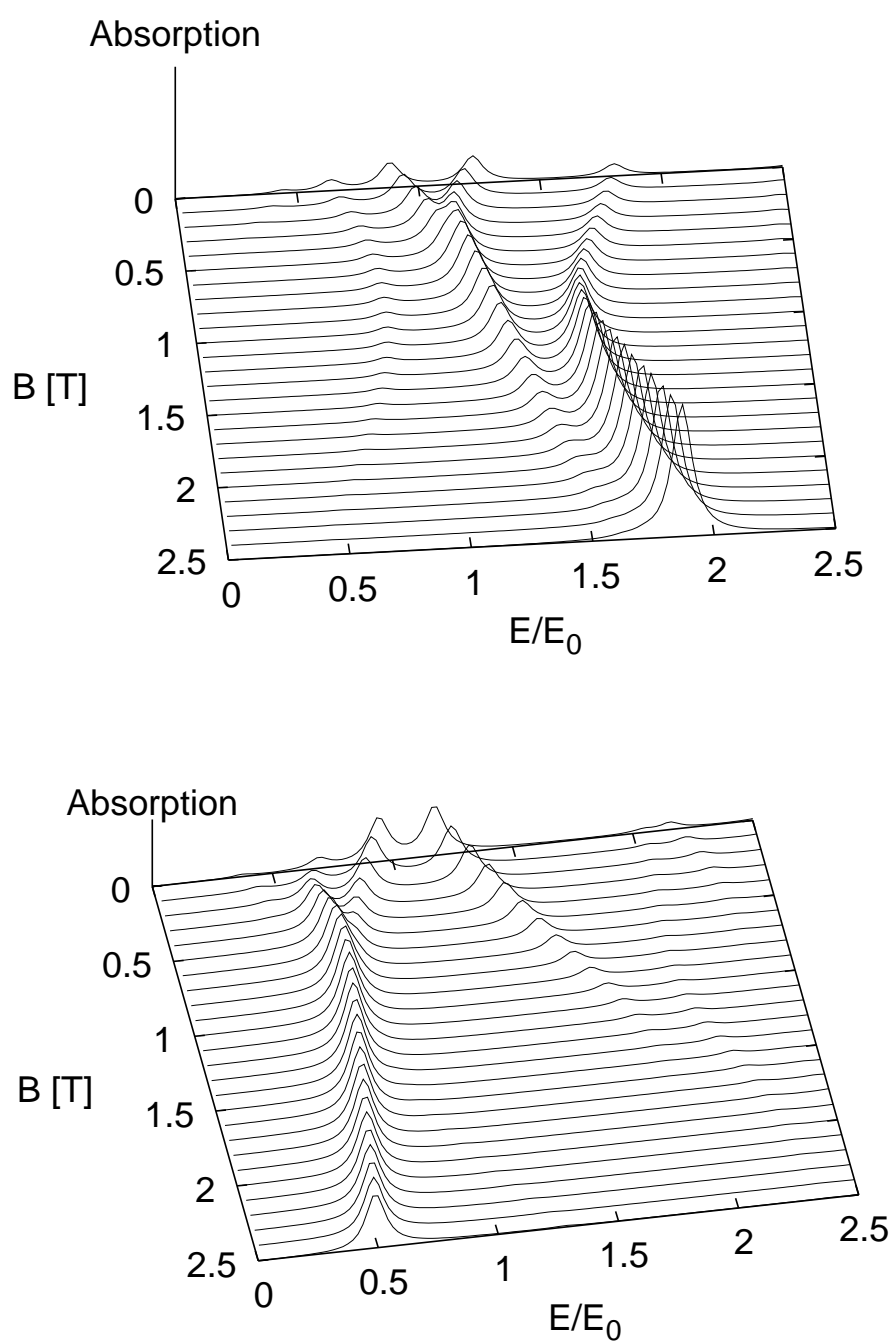

FIG. 5. The FIR absorption of a square symmetric two electron quantum dot with $\alpha_{2}=0.40$, $N_{p}=+1$ (above) and $N_{p}=-1$ (below). $E_{0}=3.37 \mathrm{meV}, T=1.0 \mathrm{~K}$. 


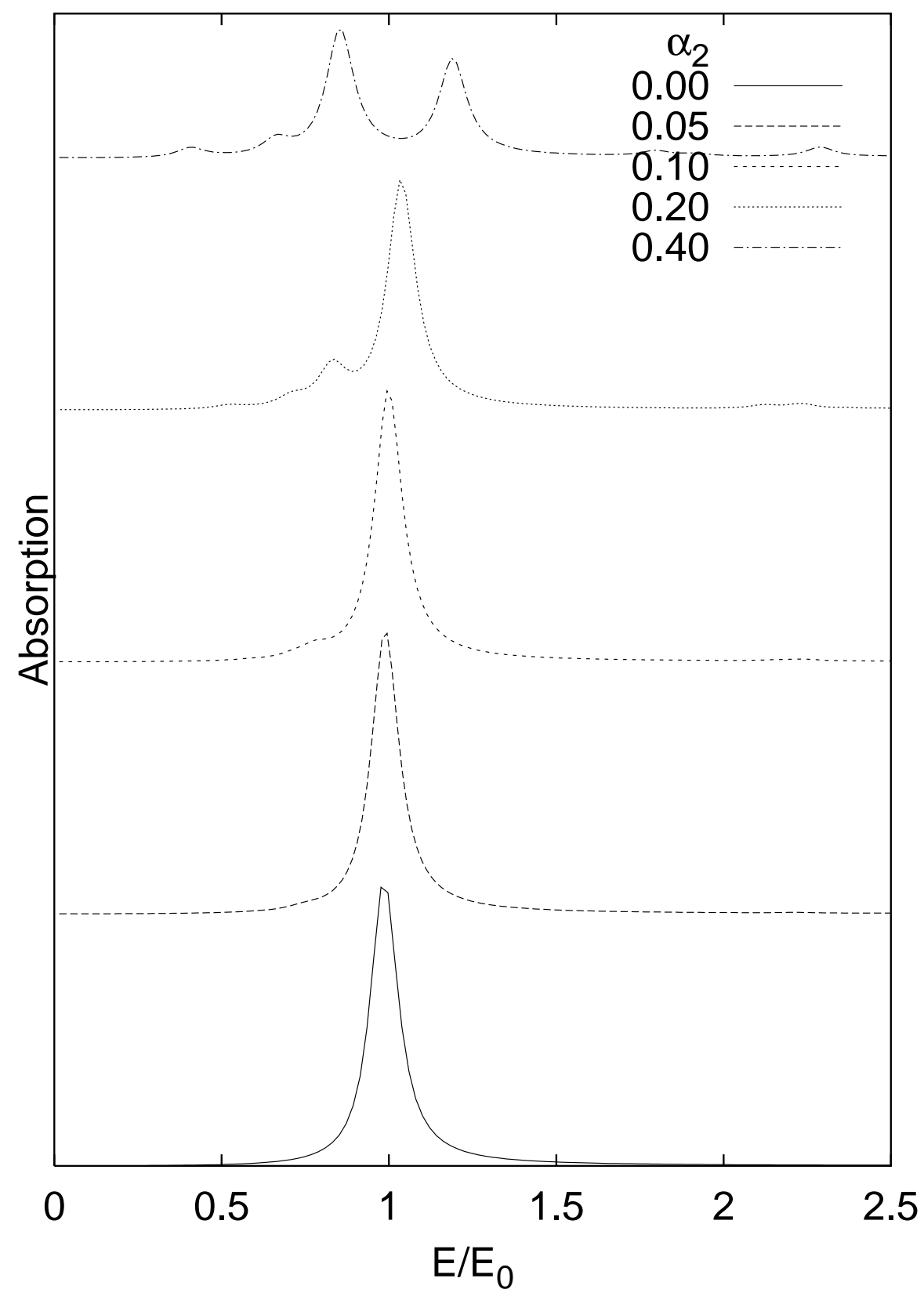

FIG. 6. The evolution of absorption peaks of a square symmetric two electron quantum dot at $B=0 T$ as $\alpha_{2}$ is increased. Influence of the square symmetric deviation from the circular parabolic confinement starts to show up in the absorption spectrum for $\alpha_{2} \geq 0.10$. 

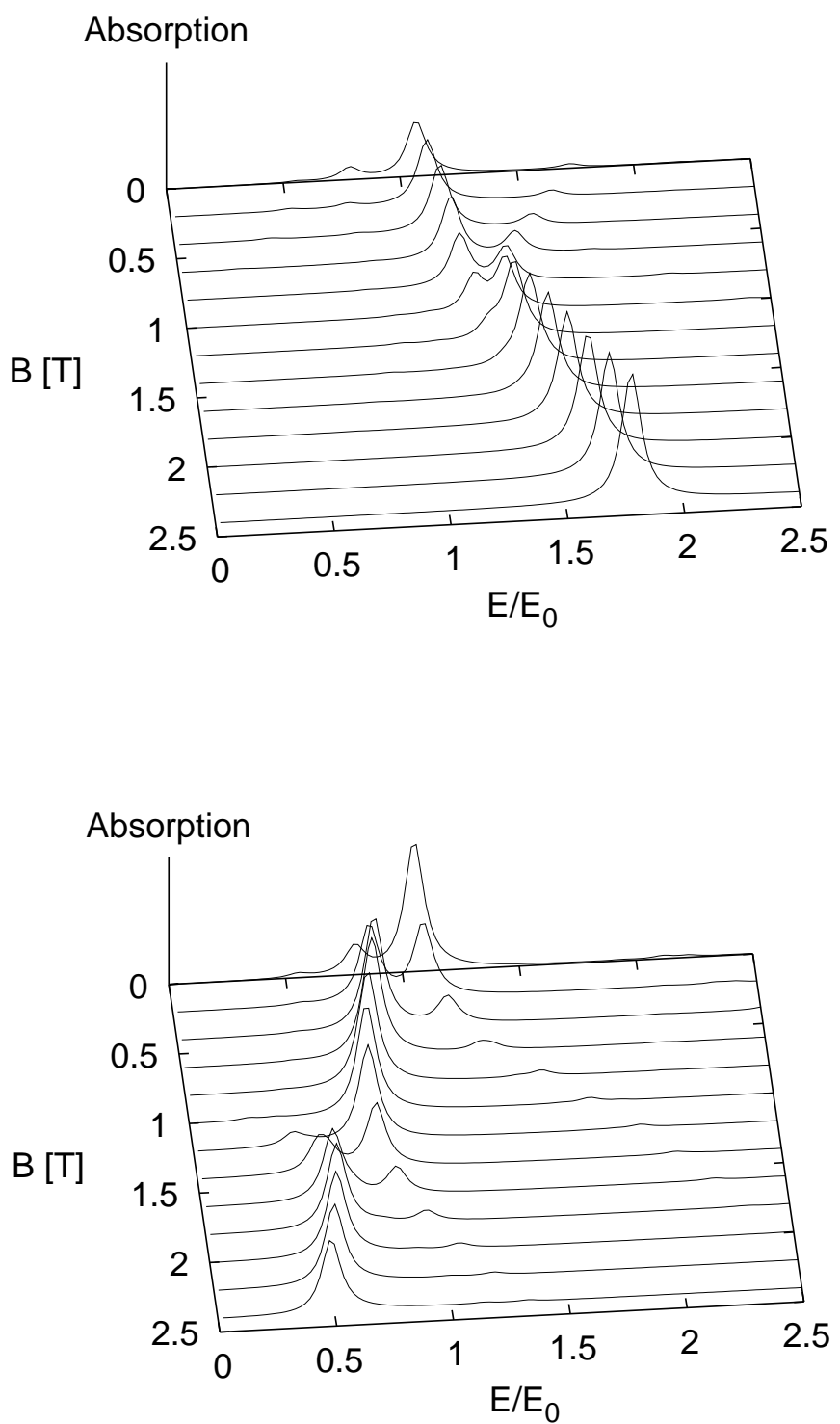

FIG. 7. The FIR absorption of a square symmetric quantum dot. Same parameters as in Fig. 1 but $N=3$. 


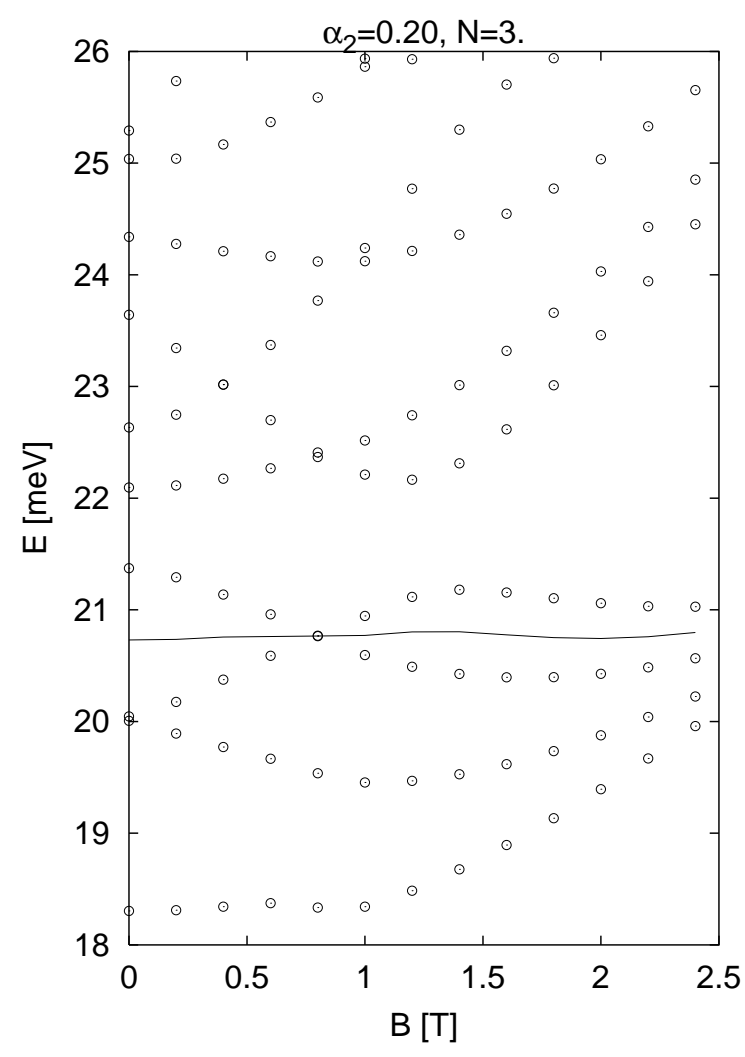

FIG. 8. The Darwin-Fock energy diagram for $N=3$ interacting electrons and $\alpha_{2}=0.20$. The chemical potential is denoted with the solid line. 

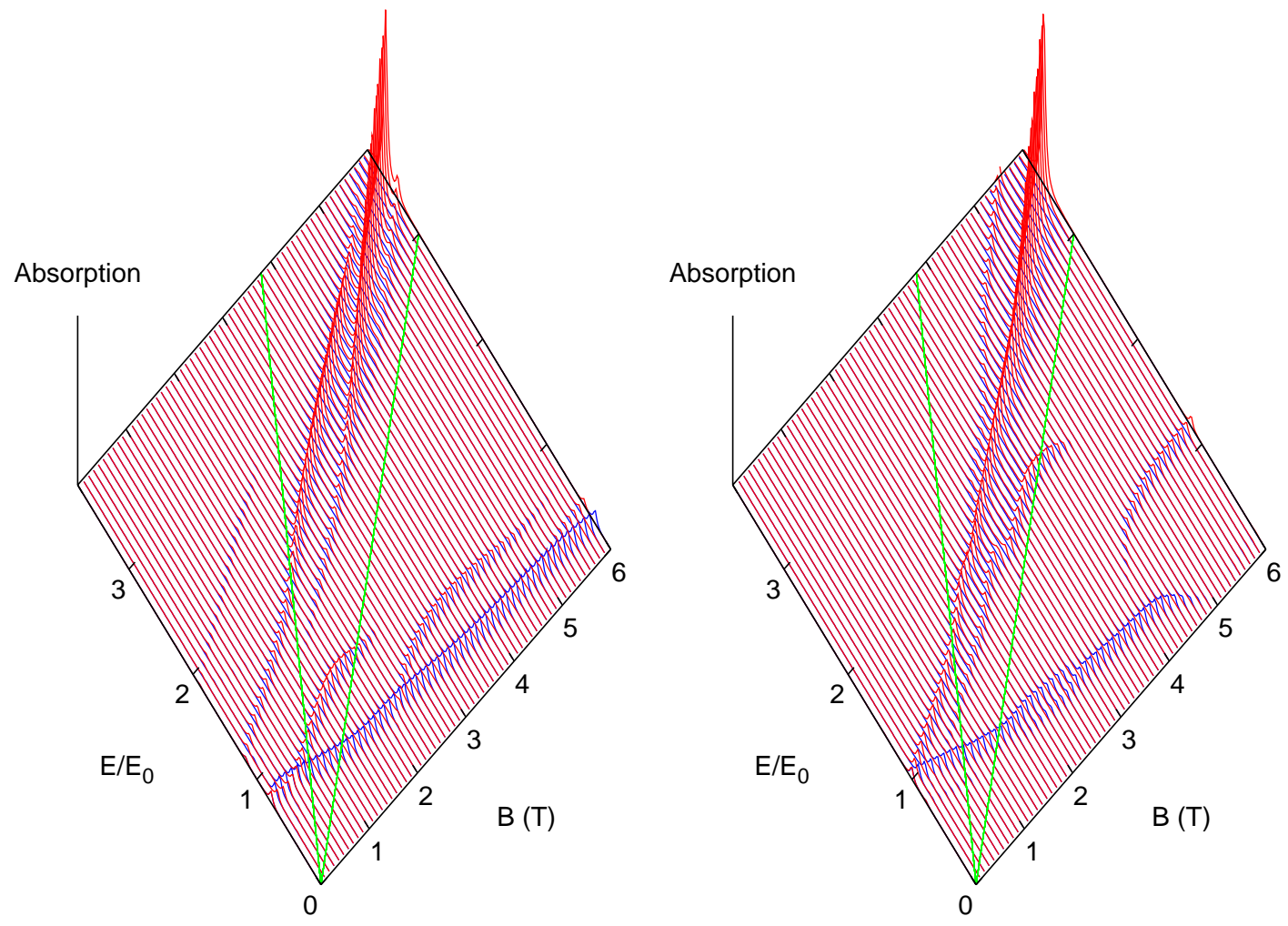

FIG. 9. The far-infrared absorption $P\left(E / E_{0}, B\right)$ in the case of $N=2$ (left), and $N=12$ (right) electrons in a circular symmetric quantum dot with the center removed. The straight lines indicate the cyclotron resonance and its first harmonic. Only the lowest branch in each case corresponds to $N_{p}=-1$ and all other branches belong to $N_{p}=+1$. $E_{0}=\hbar \omega_{0}=3.37 \mathrm{meV}, T=1.0 \mathrm{~K}$. 

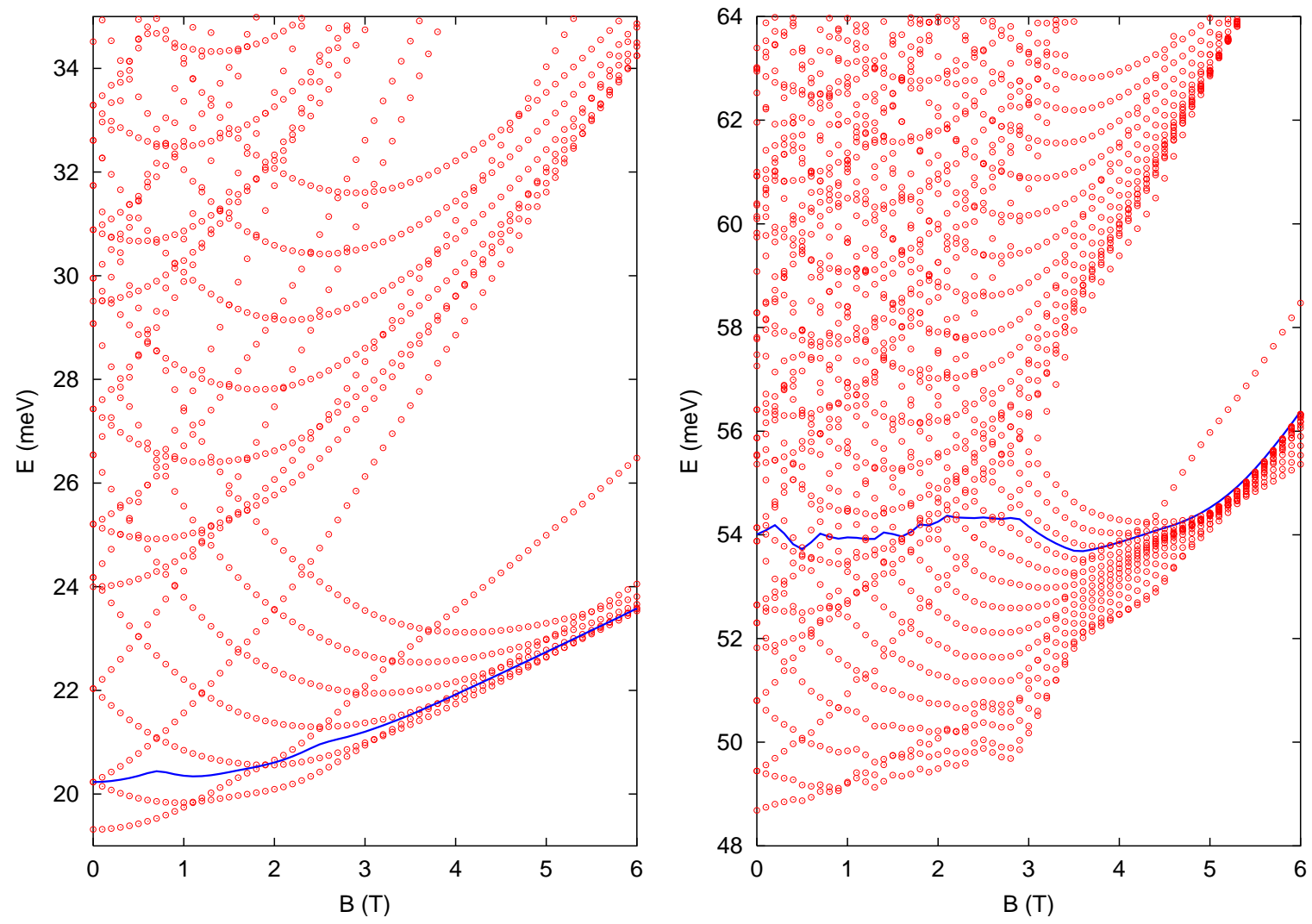

FIG. 10. The Darwin-Fock energy diagram in the case of $N=2$ (left), and $N=12$ (right) electrons in a circular symmetric quantum dot with the center removed. The chemical potential $\mu$ is indicated by the solid curve. 